Chapter 10

\title{
Evaluation of the Distortion and Unbalance Emission Levels in Electric Networks
}

\author{
Patricio Salmerón Revuelta and \\ Alejandro Pérez Vallés \\ Additional information is available at the end of the chapter \\ http://dx.doi.org/10.5772/53373
}

\section{Introduction}

Electrical disturbances have important economic consequences for the consumer and the utilities, a fact which increases from the new electricity regulatory framework. Thus, Electric Power Quality, EPQ, has now become a priority within the field of electrical engineering. Power-quality deterioration is due to transient disturbances (voltage sags, voltage swells, impulses, etc.) and steady state disturbances (harmonic distortion, unbalance, and flicker). Among these are the so-called periodic disturbances such as distortion of the voltage and current waveforms, or unbalanced three-phase systems, [1-2]. This chapter is focused specifically on harmonic and unbalance phenomena.

These quality problems have entailed the need for measuring equipment to monitor the installation at the user side such as electric power quality analyzers, [3-7]. This equipment has a great number of quantities available relating to the harmonic distortion for each phase. Each measurement is usually composed of the voltage and current total harmonic distortion (THD) index, and RMS values of the total waveforms and the fundamental component. One problem with characterization of these data is representation of harmonic distortion when the installation has very different distortion levels in each phase. There are two choices: to consider the distortion level of each phase separately, managing three times more information, or to characterize the installation distortion using a global parameter which considers the whole three-phase system, [5, 7]. The first approach is more interesting for analyzing the cause of the problems in an installation. The second is more 
suited to characterizing the distortion level of the whole installation. This point of view is adopted in this chapter.

On the other hand, it is necessary to know the responsible for the production of periodic perturbations. That is, first, identification of distortion sources, and secondly, unbalances emission. Regarding on localization of sources producing distortion two different approaches can be distinguished: a) those based on measurements taken on the point of common coupling, PCC, [9-21], b) those based one measurement taken and processed simultaneously on different metering sections placed on the line connected to the same PCC, [22-24]. This chapter is developed within the first group. Thus, the objective to localization of sources producing distortion is to measure instantaneous values of current and voltage in each of the branches of the PCC, and from these measurements, establish which consumers are responsible for generating the distortion and quantify the distortion generated by each consumer. Regarding the issue of unbalance emission, it is measurement and evaluating the negative and zero sequence currents injected by loads of unbalanced structure at the PCC.

Originally, the problem was analyzed through the harmonic power sign with the objective of knowing the sense of harmonic power flow between source and one load in distorted systems. Nowadays, it has already been established in the technical literature that an analysis of this kind does not solve the problem, [10-11]. Recently, new indices have been introduced to evaluate a specific consumer distortion and unbalance level, [12, 15, 19]. In this chapter, a comparative analysis of these indices is carried out, having as reference different practical cases. The results obtained show that, in fact, these indices can help to valuate the periodic perturbation, although none of them solve the question definitively.

More recently, it has been found that the measure of quality indices of EPQ presents additional difficulties in the presence of capacitors. The capacitor do not produce harmonic but their presence contributes to the amplification of the harmonics existing in the electrical network. The capacitor behaviour makes that the indices proposed up now to identify distortion sources fail in the presence of this element, [25]. However, in [21] has proposed a new method that resolves the situation.

The chapter is structured as follows. Second section begins with the definitions of effective voltage and current contained in the IEEE Standard 1459 and continuous with the most suited distortion and unbalance indices for three-phase four-wire networks for characterizing the quality of the waveforms present in the PCC. In third section presents the theoretical basis of the main methods to identify sources of unbalance and distortion: harmonic powers, conforming and nonconforming currents, balanced linear currents and unbalanced non-linear currents. A comparative analysis of different PQ indices based on digital simulations has been performed. The fourth section analyzes the problems that present PQ indices in the presence of installed capacitor banks for power factor correction. The fifth section describes an experimental setup for measuring the PQ indices of a three-phase nonlinear load connected to the mains. Finally, a discussion of the results and conclusions are extracted. 


\section{Assessment of harmonic distortion and unbalance in power systems}

In any system of measurement for power system, is usual to introduce a number of quantities characterizing the waveforms of voltage and current. This requiere to define the effective values of voltage, $\mathrm{V}_{\mathrm{e}}$, and current, $\mathrm{I}_{\mathrm{e}}$; here the definitions adopted in the IEEE Standard 1459-2000 (Std 1459), [26], are introduced. The definitions of these quantities are directly related to the definition of apparent power. Thus, in the 1459 Std apparent power is the maximum power that can be transmitted under ideal conditions (sinusoidal single phase or balanced three-phase sinusoidal systems) with the same impact of voltage (on the insulation and on the no-load losses) and the same impact of current (or line losses) from the PCC on the network. From this definition equivalent values of voltage and current which characterize the load impact on the power system are deduced.

\subsection{Preliminary definitions}

The explicit expression of the apparent power depends on how are characterized these voltage and current impacts. To determine the apparent power, we introduce an equivalent voltage and an equivalent current of a balanced system of positive sequence to produce the same impact on the network voltages and currents present in the system. In the following are determined equivalent quantities, effective voltage, $V_{e}$, and effective current, $\mathrm{I}_{\mathrm{e}}$.

A three-phase system consisting of an unbalanced load is supplied by a four-wire system wherein each of the lines has a resistance $r$ and the neutral conductor has a resistance $r_{n}$. The effective current value, $\mathrm{I}_{\mathrm{e}}$, is

$$
I_{e}=\sqrt{\frac{1}{3}\left(I_{a}^{2}+I_{b}^{2}+I_{c}^{2}+\rho I_{n}^{2}\right)} \quad ; \quad \rho=\frac{r_{n}}{r}
$$

The 1459 Std is $\varrho=1$. On the premises of medium and low voltage typical $\varrho=0.2-4$. Today, digital instrumentation can develop equipment that can adjust $\mathrm{Q}$ for any default.

The next step is to find an effective voltage $V_{\mathrm{e}}$. This will take into account the non load power losses in magnetic cores of transformers and insulation 'upstream' of the load. Standard means that losses (non load losses voltage-dependent), $\mathrm{P}_{\mathrm{Y}}$, which are due to the line-neutral voltages and losses (non load losses voltage-dependent), $\mathrm{P}_{\Delta}$, which are due to the line-line voltages are equal. Thus, the expression for $\mathrm{V}_{\mathrm{e}}$ :

$$
V_{e}=\sqrt{\frac{3\left(V_{a n}^{2}+V_{b n}^{2}+V_{c n}^{2}\right)+\xi\left(V_{a b}^{2}+V_{b c}^{2}+V_{c a}^{2}\right)}{9(1+\xi)}}
$$

Where $\xi$ is the power ratio, 


$$
\xi=\frac{{ }^{P}}{P_{Y}}
$$

Later, the concept of losses which depend on the voltage was abandoned in favor of whichever of loads that consume an equivalent active power, so that to determine the equivalent voltage $\mathrm{V}_{\mathrm{e}}$ is assumed that the load is formed by a group resistors connected in $\mathrm{Y}$, which consume a power $\mathrm{P}_{Y}$ and a group connected in $\Delta$, which consume a power $\mathrm{P}_{\Delta}$. In any case the St 1459 considers $\xi=1$. To a three-wire three-phase system where $1_{n}=0$, the standard recommended simplified expressions,

$$
V_{e}=\sqrt{\frac{V_{a b}^{2}+V_{b c}^{2}+V_{c a}^{2}}{9}} \quad ; \quad I_{e}=\sqrt{\frac{I_{a}^{2}+I_{b}^{2}+I_{c}^{2}}{3}}
$$

referred to as the Buchholz-Goodhue, and original works proposed by the IEEE working group on nonsinusoidal situations.

As already stated above, the definition Std 1459 takes as apparent power or effective apparent power of the system, $S_{e}$, that originally suggested by F. Buchholz in 1922 and clarified by W. M. Goodhue in 1933,

$$
\mathrm{S}_{\mathrm{e}}=3 \mathrm{~V}_{\mathrm{e}} \mathrm{I}_{\mathrm{e}}
$$

Similarly for a total active power consumed by a load, $\mathrm{P}$, the power factor is defined as,

$$
P F=\frac{P}{S_{e}}
$$

relating the minimum power loss and the current power loss.

The RMS values of the magnitudes of phase voltage and line current are determined as,

$$
\begin{aligned}
& \mathrm{V}_{\mathrm{j}}^{2}=\mathrm{V}_{\mathrm{j} 1}^{2}+\sum_{\forall \mathrm{h} \neq 1} \mathrm{~V}_{\mathrm{jh}}^{2} \\
& \mathrm{I}_{\mathrm{j}}^{2}=\mathrm{I}_{\mathrm{j} 1}^{2}+\sum_{\forall \mathrm{h} \neq 1} \mathrm{I}_{\mathrm{jh}}^{2} ; j=\mathrm{j}, \mathrm{b}, \mathrm{c}
\end{aligned}
$$

Likewise, it is desirable to split the effective voltage and current into two terms, one for the fundamental harmonic and the other for the remainder of the harmonics, 


$$
\mathrm{V}_{\mathrm{e}}^{2}=\mathrm{V}_{\mathrm{e} 1}^{2}+\mathrm{V}_{\mathrm{eH}}^{2} \quad ; \quad \mathrm{I}_{\mathrm{e}}^{2}=\mathrm{I}_{\mathrm{e} 1}^{2}+\mathrm{I}_{\mathrm{eH}}^{2}
$$

where the subscript '1 'refers to the RMS values of the fundamental component,

$$
\mathrm{V}_{\mathrm{e} 1}=\sqrt{\frac{3\left(V_{a n 1}^{2}+V_{b n 1}^{2}+V_{c n 1}^{2}\right)+\left(V_{a b 1}^{2}+V_{b c 1}^{2}+V_{c a 1}^{2}\right)}{18}} \quad ; \quad \mathrm{I}_{\mathrm{e} 1}=\sqrt{\frac{\mathrm{I}_{\mathrm{a} 1}^{2}+\mathrm{I}_{\mathrm{b} 1}^{2}+\mathrm{I}_{\mathrm{c} 1}^{2}+\mathrm{I}_{\mathrm{n} 1}}{3}}
$$

and the subscript 'H' refers to the whole of the harmonic components other than the fundamental,

$$
\mathrm{V}_{\mathrm{eH}}=\sqrt{\sum_{\mathrm{h} \neq 1} \frac{3\left(V_{a n h}^{2}+V_{b n h}^{2}+V_{c n h}^{2}\right)+\left(V_{a b h}^{2}+V_{b c h}^{2}+V_{c a h}^{2}\right)}{18}} ; \mathrm{I}_{\mathrm{eH}}=\sqrt{\sum_{\mathrm{h} \neq 1}\left(\frac{\mathrm{I}_{\mathrm{ah}}^{2}+\mathrm{I}_{\mathrm{bh}}^{2}+\mathrm{I}_{\mathrm{ch}}^{2}+\mathrm{I}_{\mathrm{nh}}^{2}}{3}\right)}
$$

\subsection{Power quality indexes}

The use of effective voltage and current values helps characterize the harmonic content of the three-phase system by using the so-called total harmonic distortion (THD) rates of voltage and current, [4]. Thus, for any phase $\phi(a, b, c)$ the voltage total harmonic distortion is,

$$
V T H D_{\phi}=\sqrt{\frac{V_{\phi}^{2}-V_{\phi_{1}}^{2}}{V_{\phi_{1}}^{2}}}=\sqrt{\frac{V_{\phi_{2}}^{2}+V_{\phi_{3}}^{2}+V_{\phi_{4}}^{2}+\ldots+V_{\phi_{N}}^{2}}{V_{\phi_{1}}^{2}}}
$$

and the current total harmonic distortion is,

$$
I T H D_{\phi}=\sqrt{\frac{I_{\phi}^{2}-I_{\phi_{1}}^{2}}{I_{\phi_{1}}^{2}}}=\sqrt{\frac{I_{\phi_{2}}^{2}+I_{\phi_{3}}^{2}+I_{\phi_{4}}^{2}+\ldots+I_{\phi_{N}}^{2}}{I_{\phi_{1}}^{2}}}
$$

where the second subscript represents the order of the harmonic. These indices THD, harmonic content of the waveform are compared with the fundamental harmonic. This is a definition in the standard IEC 61000 and is common in commercial network analyzers. However, a second definition is introduced where the harmonic content is compared to the RMS value of the waveform, [8]. Here we refer to this factor as the rate of total demand distortion, TDD. For voltage, 


$$
V T D D_{\phi}=\sqrt{\frac{V_{\phi}^{2}-V_{\phi_{1}}^{2}}{V_{\phi}^{2}}}=\sqrt{\frac{V_{\phi_{2}}^{2}+V_{\phi_{3}}^{2}+V_{\phi_{4}}^{2}+\ldots+V_{\phi_{N}}^{2}}{V_{\phi}^{2}}}
$$

and to the current,

$$
\operatorname{ITDD}_{\phi}=\sqrt{\frac{I_{\phi}^{2}-I_{\phi_{1}}^{2}}{I_{\phi}^{2}}}=\sqrt{\frac{I_{\phi_{2}}^{2}+I_{\phi_{3}}^{2}+I_{\phi_{4}}^{2}+\ldots+I_{\phi_{N}}^{2}}{I_{\phi}^{2}}}
$$

The values provided by THD and TDD factors are often very similar to waveforms with low distortion, yet their differences are more significant for high distortions. THD factors indicate a very high value, even infinite, when the waveform has a very small or zero value of the fundamental harmonic. Moreover, TDD values are always below $100 \%$ and this is somewhat larger errors in measurement instrumentation versus normal situations THD distortion.

The distortion characterization of the global systems requires extending the definition of harmonic distortion rates to three-phase systems. The rate of three-phase voltage total harmonic distortion, $\mathrm{VTHD}_{3 \phi}$, and the rate of three-phase current, $\mathrm{ITHD}_{3 \phi}$, is defined as,

$$
\operatorname{ITHD}_{3 \phi}=\sqrt{\frac{I_{e}^{2}-I_{e 1}^{2}}{I_{e 1}^{2}}} ; \operatorname{VTHD}_{3 \phi}=\sqrt{\frac{V_{e}^{2}-V_{e 1}^{2}}{V_{e 1}^{2}}}
$$

Similarly, is possible to define the three-phase total demand distortion of voltage, $\operatorname{VTDD}_{3 \phi}$ and the three-phase total demand distortion of current, $\operatorname{ITDD}_{3 \phi}$,

$$
\operatorname{ITDD}_{3 \phi}=\sqrt{\frac{I_{e}^{2}-I_{e 1}^{2}}{I_{e}^{2}}} ; \operatorname{VTDD}{ }_{3 \phi}=\sqrt{\frac{V_{e}^{2}-V_{e 1}^{2}}{V_{e}^{2}}}
$$

According to their definition, the three-phase total demand distortion indices measure the lack of conformity of the line voltage and current waveforms with respect to sinusoidal waveforms, because of the harmonic content; without considering possible unbalances.

It is important to point out that these new indices may be calculated in a simple way with the harmonic measurements available from a commercial device. Usually, a power quality Analyzer supplies THD values, RMS values and fundamental component RMS values corresponding to each harmonic in each phase. So, the defined indices can be calculated for the measurements of each phase. For instance, the three-phase distortion indices can be calculated by means of the following expressions: to the voltage, 


$$
V T D D_{3 \phi}=\sqrt{V T H D_{a}^{2} \frac{V_{a_{1}}^{2}}{V_{e}^{2}}+V T H D^{2} \frac{V_{b_{1}}^{2}}{V_{e}^{2}}+V \operatorname{THD}^{2} \frac{V_{c_{1}}^{2}}{V_{e}^{2}}+V \operatorname{THD}_{n}^{2} \frac{V_{n_{1}}^{2}}{V_{e}^{2}}}
$$

and to the current

$$
\operatorname{ITDD}_{3 \phi}=\sqrt{\operatorname{ITHD}_{a}^{2} \frac{I_{a_{1}}^{2}}{I_{e}^{2}}+I T H D_{b}^{2} \frac{I_{b_{1}}^{2}}{I_{e}^{2}}+I T H D_{c}^{2} \frac{I_{c_{1}}^{2}}{I_{e}^{2}}+I T H D_{n}^{2} \frac{I_{n_{1}}^{2}}{I_{e}^{2}}}
$$

To evaluate the unbalance conditions, a reference waveform may be established [2, 23]. In this paper a reference voltage waveform and a reference current waveform are defined in a similar way: they are sinusoidal and positive sequence waveforms whose RMS values, $V_{\mathrm{e} 1+}$ and $\mathrm{I}_{\mathrm{e} 1+}$, are the effective voltage and current defined in similar form as (16), respectively.

$$
V T D D_{3 \phi}^{+}=\sqrt{\frac{V_{e}^{2}-V_{e 1+}^{2}}{V_{e}^{2}}} ; \operatorname{ITDD}{ }_{3 \phi}^{+}=\sqrt{\frac{I_{e}^{2}-I_{e 1+}^{2}}{I_{e}^{2}}}
$$

These new factors act as indices of non-conformity of voltage and current waveforms with respect to sinusoidal waveforms of positive phase sequence of voltage and current, respectively. A comparison between the values obtained by applying the expressions (16) and (19) would determine whether the periodic disturbances are mainly due to the presence of distortion or the presence of unbalance. Finally, in [8] a weighted distortion index has been proposed. Their calculation is based on the RMS values of each phase. However, usually, the three-phase power system is not balanced. So, the power transferred by each phase differs considerably from the other phases. It affects the information supplied by the current indices in a relevant manner. For that reason, the definition of a new distortion index which takes this new effect into account may be interesting.

However, the indices defined in (17), (18), and (19) are not appropriated for establishing responsibility, between customer and supplier, for the lack of electric power quality.

\section{Localization of sources producing distortion}

The identification of the loads they produce distortion has been addressed by analyzing the direction of active harmonic power flow. Therefore, this section begins with the analysis of active harmonic power. Other indices are introduced with the aim of overcoming the limitations of the method of harmonic powers. Several practical cases using a simulation environment will be studied and a comparative analysis is performed. 


\subsection{Analisys of active harmonic powers}

In a first approximation to the problem, balanced three-phase system are considered with several non-linear loads connected to PCC. Figure 1 shows the equivalent single-phase circuit, which includes $n+1$ branch, $n$ corresponding to the loads and 1 to the supply. The grid is represented by the Thevenin circuit constituted by a voltage source Vs in series with inductive impedance Zs. Certainly, PCC in figure 1 represents a bus of a distribution system.

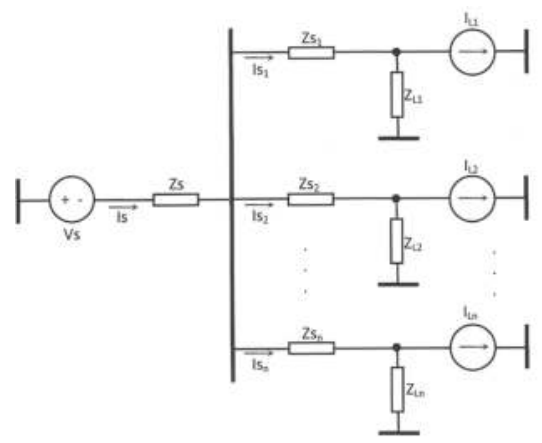

Figure 1. Non linear balanced three-phase system equivalent circuit.

Consumers are supplied by single impedances, where each load is modelled as impedance $\mathrm{Z}_{\mathrm{Lj}}$ connected in parallel to a harmonic source $\mathrm{I}_{\mathrm{Lj}}$, for $\mathrm{j}$ branch. Due to the system topology, it is necessary the measurement of voltage and current in each branch connected to the PCC. From these measurements, the consumers responsible of distortion are established. Besides, distortion generated by each one is quantified. Thus, figure 1 can be reduced to figure 2 .

Consumers are supplied by single impedances, where each load is modelled as impedance $\mathrm{Z}_{\mathrm{Lj}}$ connected in parallel to a harmonic source $\mathrm{I}_{\mathrm{Lj}}$, for $\mathrm{j}$ branch. Due to the system topology, it is necessary the measurement of voltage and current in each branch connected to the PCC. From these measurements, the consumers responsible of distortion are established. Besides, distortion generated by each one is quantified. Thus, figure 1 can be reduced to figure 2 .

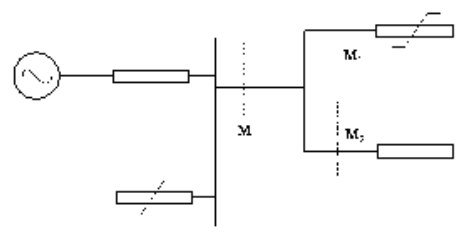

Figure 2. Sections of measurements in a power system simplified diagram with two loads connected to PCC.

From now on, diagram presented in figure 2 is considered as reference. There is a PCC where a sinusoidal voltage source is connected through source impedance. Besides, several 
linear and nonlinear loads are connected. Sections of measurements on M, M1 and M2 are considered.

A non-linear or time-varying load (measuring section M1) and linear load (measuring section M2) are considered to M. In M, bus voltage is non-sinusoidal due to the simultaneous effects of distorted caused by non-linear loads upstream and downstream $\mathrm{M}$. The analysis of power systems which include non-linear loads can be carried out two subsystems. There is a power flow between them corresponding to each harmonic, [1, 9]. The system analysis in frequency domain needs the consideration of an equivalent circuit for each relevant harmonic. Thus, the system is simplified through a Thevenin equivalent circuit from the measuring section: a nonsinusoidal Thevenin voltage and frequency dependent Thevenin equivalent impedance.

In general, there are harmonics common to Thevenin voltage and current incoming non-linear loads. So, for each common harmonic there is a harmonic active power value corresponding to grid and consumer. Its sign depends on the subsystem responsible of the prevalent contribution. According to this, a harmonic analysis can not identify the distortion source from the active powers addition, but it only can identify the source which presents the prevalent contribution. It is because both harmonic currents as harmonic powers are due to the addition of two opposite contributions. Nevertheless, harmonic active and reactive powers measurements were the approach mainly adopted to identify the pollution generated by the consumer to the supply waveform quality.

\subsubsection{An index to identify distorted loads based in harmonic powers}

The analysis presented in above section suggests the necessity of finding an index which allows the evaluation of distortion generated by a specific load in the supply voltage system. According to this approach, the index should be based on the measurements of harmonic active powers corresponding to each load is needed. Several proposals within this approach have appeared in last years. Among them, named harmonic phase index, $\xi_{\mathrm{HPI}}$ [15], defined as follows. A 3 n current vector I is introduced where $\mathrm{n}$ is the maximum harmonic order considered. Vector I is built with RMS values of each phase of each harmonic load current. This is broken in two components, $\mathrm{I}_{\mathrm{S}}$ and $\mathrm{I}_{\mathrm{L}}$ whose elements corresponding to each harmonic are defined as follows;

$$
\begin{gathered}
I_{S k}=\left\{\begin{array}{lll}
0 & \text { si } & P_{k} \leq 0 \\
I_{k} & \text { si } & P_{k} \succ 0
\end{array}\right. \\
I_{L k}=\left\{\begin{array}{lll}
0 & \text { si } & P_{k} \geq 0 \\
I_{k} & \text { si } & P_{k} \prec 0
\end{array}\right.
\end{gathered}
$$

The harmonic phase index here introduced is lightly different from the presented in [15], 


$$
u(t)=\sum_{k=1}^{n} \sqrt{2} U_{k} \sin \left(k \omega_{1} t+\theta_{k}\right) \quad ; \quad i(t)=\sum_{k=1}^{n} \sqrt{2} I_{k} \sin \left(k \omega_{1} t+\phi_{k}\right)
$$

This index has the following significant characteristics for the purpose for which it is introduced. First, it is defined from the ratio of current RMS values that are the actual cause of disturbances generated by loads in the grid. Second, different harmonic values are not added, but in quadratic sum way. It avoids mutual cancellation between different harmonics.

\subsection{Conforming and non-conforming currents}

In [12], authors distinguish two kinds of loads, conforming loads and non-conforming loads. A conforming load does not cause a change in voltage waveform distortion or in symmetry of the phases. Any other load that changes the voltage waveform or symmetry is a non-conforming load. Thus, from the harmonic point of view, the author considers that a conforming load presents a current collinear to the voltage. A typical load will be constituted by a conforming part and a non-conforming part which may be modelled in a simple way by means of two parallel elements. Current measured in the input $i(t)$ will be the sum of currents incoming to the conforming part $i_{n}(\mathrm{t})$ and the non-conforming part $i_{d}(\mathrm{t})$. The conforming current is the part of the current that presents the same distortion level as supply voltage. The rest of current is the non wished part of current; the incoming in non-conforming load. The split of power flow is obtained from the components established by the current.

Voltage and current measured in load terminals are expressed as the addition of fundamental harmonic and other components multiple of fundamental.

$$
\mathbf{Y}\left(j k w_{1}\right)=\left|\mathbf{Y}\left(j \omega_{1}\right)\right| \angle k\left(\varphi_{1}-\theta_{1}\right) \quad ; \quad \mathbf{Y}\left(j \omega_{1}\right)=\left|\frac{\mathbf{I}_{\mathbf{1}}}{\mathbf{V}_{\mathbf{1}}}\right| \angle\left(\varphi_{1}-\theta_{1}\right)
$$

The conforming current presents the same variation as voltage waveform and its phase may be lower or higher. On the other hand, due to the fact that the load can not generate power at fundamental frequency, conforming current evolves the complete fundamental active and reactive powers. Conforming current at fundamental frequency $I_{n 1}$ is equal to the total current at fundamental frequency, $\mathrm{I}_{1}$. The rest of components to the conforming current for different frequencies are proportional to the corresponding harmonic voltage components. The proportional constant is a complex ratio:

$$
\begin{aligned}
& i_{n}(t)=\sum_{k=1}^{n} \frac{I_{1}}{V_{1}} \sqrt{2} V_{k} \sin \left(k \omega_{1} t+\theta_{k}+k\left(\phi_{1}-\theta_{1}\right)\right) \\
& i_{d}(t)=i(t)-i_{n}(t)
\end{aligned}
$$


Expressions in time domain for each line current are:

$$
\mathrm{NC}=\frac{\mathrm{I}_{\mathrm{d}}}{\mathrm{I}} \times 100(\%)
$$

Where $\mathrm{V}_{1}$ and $\mathrm{I}_{1}$ are fundamental voltage and current RMS values in PCC, $\theta_{1}$ and $\phi_{1}$ are voltage and current fundamental phases, $\theta_{k}$ is the $\mathrm{k}$ order harmonic phase of voltage and $i(\mathrm{t})$ is the load current. The index of distortion proposed en [12] is the non collinear index, NC,

$$
\begin{aligned}
& \left|\mathbf{Z}_{1 a}\right|=\frac{V_{1 a}}{I_{1 a}} \\
& \angle \mathbf{Z}_{1 a}=\left|\theta_{1 a}-\phi_{1 a}\right|=\varphi_{1 a}
\end{aligned}
$$

where $\mathrm{I}_{\mathrm{d}}$ is $i_{d}(\mathrm{t})$ RMS value.

\subsection{Linear and nonlinear current}

The problem of separating the contributions to the distortion of the supply and the consumer was approached was reasoned in [19] as follows. The deterioration of EPQ due to harmonics can be caused simultaneously in several points of network. Voltage and current waveforms measured in PCC are due to the combined effect of several polluting equipment connected in different places in the network. It is possible the determination of the specific load contribution to harmonic distortion, whereas all other is considered in the supply side which includes the rest of loads. The first step is fixing the ideal load conditions. Any load which shows a linear and balanced behavior represents and ideal load condition. In fact, if specific load is balanced and linear, the supply is the only responsible of harmonic distortion in PCC. Initially, it is necessary the identification of waveform of incoming current if an equivalent linear and balanced load is presented instead of the actual incoming load current. This load can be defined as the linear load that requires a fundamental active power equal to the fundamental active power actually flow by PCC. It requires a distorted current but it is not responsible of the distortion.

In order to model the three-phase balanced linear ideal load, three identical RL branches have been considered. Through the evaluation of R, L parameters, the part of load that represents the equivalent ideal load can be identified. So, from the consumer side, it is always possible the estimation of the linear load. This part of the actual load requires a balanced and linear which constitute an ideal current and represents one component of the load current in PCC. If ideal current is almost total current, the load is not responsible of distortion problems.

Equivalent balanced and linear load parameters $\mathrm{R}$ and $\mathrm{L}$ are estimated in phase ' $\mathrm{a}$ ' according to the procedure indicated as follow, (the same method can be applied to phases ' $b$ ' and 
' $c$ '). If phase ' $a$ ' equivalent linear impedance is named by $\mathrm{Z}_{1 a}$, the R-L series circuit at fundamental frequency is:

$$
\begin{aligned}
& R_{a}=\left|\mathbf{Z}_{1 a}\right| \cos \varphi_{1 a} \\
& X_{1 a}=\left|\mathbf{Z}_{1 a}\right| \operatorname{sen} \varphi_{1 a} \\
& L_{a}=\frac{X_{1 a}}{2 \pi f_{1}}
\end{aligned}
$$

where $\mathrm{V}_{1 \mathrm{a}}$ and $\mathrm{I}_{1 \mathrm{a}}$ are voltage and current RMS values of fundamental frequency, respectively, in PCC, and $\theta_{1 a}$ and $\phi_{1 a}$ are phase angle of those magnitudes. So,

$$
X_{k a}=2 \pi k f_{1} L_{a}, \quad k=1,2, \cdots n
$$

where $X_{1 a}$ represents reactance of R-L series combination at fundamental frequency, $f_{1}$, and $\mathrm{R}_{\mathrm{a}}$ and $\mathrm{L}_{\mathrm{a}}$ are the corresponding parameters. Reactance values to voltage harmonic components are,

$$
\begin{aligned}
& \left|\mathbf{Z}_{\mathbf{k} a}\right|=\sqrt{R_{a}^{2}+X_{k a}^{2}} \\
& \varphi_{k a}=\operatorname{tag}^{-1} \frac{X_{k a}}{R_{a}}, \quad k=1,2, \cdots n
\end{aligned}
$$

The skin effect is neglected, that is, the resistance is assumed to be constant for all frequencies, and hence,

$$
i_{L a}(t)=\sum_{k=1}^{n} \frac{\mathrm{V}_{k a}}{\left|\mathbf{Z}_{\mathbf{k} a}\right|} \sqrt{2} \operatorname{sen}\left(2 \pi k f_{1} t+\left(\theta_{k a}-\varphi_{k a}\right)\right)
$$

where $\mathrm{n}$ is the most significant order of harmonic. As a consequence, current in the ideal linear load in phase ' $a$ ' is,

$$
i_{n L a}(t)=i_{a}(t)-i_{L a}(t)
$$

This is the equivalent linear current and the supply system is the only responsible of its distortion. The difference between measured current and calculated ideal current is defined as non-linear current: 


$$
N L=\frac{I_{n L a}}{I_{a}} \times 100(\%)
$$

The non-linear current indicates how much the actual load current in phase 'a' differs from the ideal linear load in terms of harmonic distortion. Thus, an index of non-linear current is defined with the intention to provide reliable information about distortion caused by the consumer:

$$
\mathrm{V}_{+}=\frac{1}{3}\left(\mathrm{~V}_{\mathrm{a}}+\mathrm{aV}_{\mathrm{b}}+\mathrm{a}^{2} \mathrm{~V}_{\mathrm{c}}\right)
$$

where $I_{n L a}$ and $I_{a}$ are the RMS values corresponding to non-linear and actual current, respectively.

\subsection{Practical cases: Digital simulations}

Harmonic powers and indices for assessing the harmonic distortion introduced in the previous sections have been applied to the system shown in Figure 3. This is a balanced system consisting of two AC / DC converters controlled firing angles $25^{\circ}$ (Rectifier 1 ) and $45^{\circ}$ (Rectifier 2).

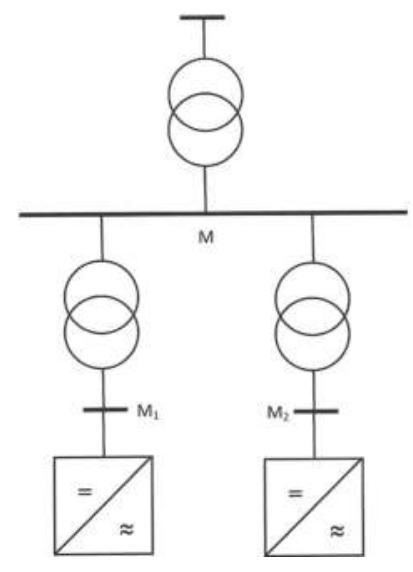

Figure 3. Circuit topology for the practical cases.

\subsubsection{Case 1: Sinusoidal voltage}

In the first situation has been considered a sinusoidal Thevenin voltage upstream of $\mathrm{M}$ and network parameters given by $\mathrm{R}=0.005 \Omega$ and $\mathrm{L}=9,5 \times 10^{-5} \mathrm{H}$. Under these conditions, the measuring point $\mathrm{M}$, the VTDD is $15.06 \%$. 
Table 1 lists the powers of the main harmonics in the three measurement points $M, M_{1}, M_{2}$, Figure 3. The same table includes the values of total active power, fundamental and harmonic power in the three sections of measurement.

\begin{tabular}{cccc}
\hline & Two loads & Load 1 & Load 2 \\
\hline P1 (W) & $1.9129 \cdot 10^{5}$ & $1.1377 \cdot 10^{5}$ & $7.7512 \cdot 10^{4}$ \\
\hline P5 (W) & $-3.4427 \cdot 10^{2}$ & $-1.2706 \cdot 10^{3}$ & $9.2632 \cdot 10^{2}$ \\
\hline P7 (W) & $-1.2238 \cdot 10^{1}$ & $-2.1575 \cdot 10^{1}$ & $9.3370 \cdot 10^{0}$ \\
\hline P11 (W) & $3.7115 \cdot 10^{1}$ & $1.5520 \cdot 10^{2}$ & $-1.1809 \cdot 10^{2}$ \\
\hline P13 (W) & $1.1830 \cdot 10^{2}$ & $2.7183 \cdot 10^{2}$ & $-1.6761 \cdot 10^{2}$ \\
\hline P17 (W) & $4.7315 \cdot 10^{1}$ & $2.7183 \cdot 10^{2}$ & $-2.2452 \cdot 10^{2}$ \\
\hline PT (W) & $1.9127 \cdot 10^{5}$ & $1.1365 \cdot 10^{5}$ & $7.7620 \cdot 10^{4}$ \\
\hline PH (W) & $-1.4683 \cdot 10^{1}$ & $-1.2237 \cdot 10^{2}$ & $1.0769 \cdot 10^{2}$ \\
\hline
\end{tabular}

Table 1. Active powers of the main harmonics and global results for the case 1.

The total harmonic power (point M) is $-14.68 \mathrm{~W}$ in the direction of power flow from consumer to network. This is because the harmonic powers of certain orders are negative and larger absolute value than those of the harmonics with positive powers. Harmonic power measuring section $\mathrm{M}$ becomes a indicator of consumption nonlinear produced downstream of $\mathrm{M}$. However, the presence of two non-linear loads of different consumption changes the situation and the harmonic power fail when their values are obtained in the measuring sections $M_{1}$ and $M_{2}$. Indeed, in the section of the $\mathrm{M}_{2}$ measure harmonic power $\mathrm{PH}_{2}$ is $107.69 \mathrm{~W}$ in the direction from network to load, while in $\mathrm{M}_{1}$, the harmonic power $\mathrm{PH}_{1}$ is $-12.37 \mathrm{~W}$ in the direction from load to network. That is, the method of direction of power flow, when more than one source of distortion, determines the prevalent power for each harmonic, corresponding to the power flows in opposite directions, load to network versus network to load, into measuring point. Therefore, when more than one source of distortion, it is not possible to reliably identify the responsible of the disturbance through only the harmonic power. Table 2 presents four indices to assess the distortion. The first ITDD characterized the harmonic content of the current waveforms. The remaining three are defined to identify sources of distortion.

\begin{tabular}{cccc}
\hline & Two loads & Load 1 & Load 2 \\
\hline ITDD & 23.59 & 31.14 & 37.68 \\
\hline HPI & 22.08 & 26.37 & 16.70 \\
\hline NC & 29.76 & 34.92 & 41.30 \\
\hline NL & 25.92 & 33.17 & 38.97 \\
\hline
\end{tabular}

Table 2. Current Total Demand Distortion, Harmonic Phase Index, Non Collinear Index and Non Linear Index values for the case 1. 
HPI index account the current values prevailing at the measuring point. HPI indices in the three measuring points indicate currents prevalent from consumer to source direction, and are suitable for assessing the contribution of both loads. The same table 2 also includes the indices NC and NL. For measures in M, the three indices have consistent values. However, for each individual load, the HPI index assigns greater responsibility for the distortion to the load 1 before to the load 2, while NC and NL rates assigned greater responsibility to the load 2 before to the load 1 . The values obtained by the latter two are consistent.

\subsubsection{Case 2: Non-sinusoidal voltage}

Tables 3 and 4 present the results for the same system of figure 3 but this time the TDD voltage at point $\mathrm{M}$ is $12.67 \%$. In this case, the Thevenin voltage network is not sinusoidal but includes harmonics of order 5 and 7 . Harmonic powers $P_{H}$ are positive in all measurement points.

\begin{tabular}{cccc}
\hline & Two load & Load 1 & Load 2 \\
\hline PT(W) & $1.54371 \cdot 10^{5}$ & $9.7743 \cdot 10^{4}$ & $5.6623 \cdot 10^{4}$ \\
\hline P1 (W) & $1.4796 \cdot 10^{5}$ & $9.5058 \cdot 10^{4}$ & $5.2907 \cdot 10^{4}$ \\
\hline PH (W) & $6.4011 \cdot 10^{3}$ & $2.6845 \cdot 10^{3}$ & $3.7166 \cdot 10^{3}$ \\
\hline
\end{tabular}

Table 3. Active powers of the main harmonics and global results for the case 2 .

The indices NC and NL, Table 4, have values consistent with those obtained in the previous situation. But not so with HPI indices showing less stability against variations in network conditions. This time the HPI index shows an allocation of responsibility for the distortion between charges 1 and 2 contrary to case 1 . By contrast, the results table further shows adequate stability of NL and NC indices versus distortion variations network.

\begin{tabular}{cccc}
\hline & Two loads & Load 1 & Load 2 \\
\hline ITDD & 23.62 & 30.78 & 38.25 \\
\hline HPI & 6.78 & 2.28 & 20.83 \\
\hline NC & 30.67 & 35.57 & 42.97 \\
\hline NL & 25.46 & 33.00 & 38.82 \\
\hline
\end{tabular}

Table 4. Current Total Demand Distortion, Harmonic Phase Index, Non Collinear Index and Non Linear Index values in the case 2 .

\section{On the measurement of PQ indices in the presence of capacitor bank}

In $[21,25]$ has shown experimentally that the presence of equipment power factor compensation capacitors based on amplified the distortion existing in the network. Moreover, as 
discussed later, the techniques proposed for assigning responsibility for harmonic distortion penalize capacitors like nonlinear loads. This is in clear contradiction with the vast majority of standards that require the use of capacitors for power factor correction to the fundamental frequency.

Indeed, an electronic load of the type power converter absorbs a current obtained through the sudden switching of electronic devices according to the on-off states. This current produces voltage drops in the inductive impedance of the grid, causing voltage peaks in the voltage waveform at the PCC. As a result, the current drawn by the linear load has a peak and RMS values higher because the load capacitors which tend to emphasize the high harmonics at the facility. This would be the case, for example, a set of discharge lamps (compensated) connected to the same PCC that a nonlinear load. The simplified equivalent circuit comprises an ohmic-inductive branch and a capacitive branch. High current harmonics are almost completely absorbed by the capacitive branch, since its impedance at these frequencies is very small compared with that of the other branch. This contributes to the increased distortion of the current absorbed by the linear load. Therefore, although the capacitors do not introduce new harmonics on the network, if they can dramatically amplify existing distortion produced by nearby non-linear loads.

To illustrate the situation has been considered a system consisting of a six-pulse rectifier controlled with a consumption of $91.77 \mathrm{~kW}$ in parallel with a line load consisting of $4 \Omega$ resistor in parallel with a capacitor of $0.1 \mathrm{mF}$. The figure a) shows the waveform of the phase voltage at the PCC and figure b) the waveform of the current drawn by the RC load.

This has consequences for the effectiveness of the various indices introduced to identify sources of distortion. The situation is illustrated through two practical cases of simulation.

\subsection{Case 1: Inductive and capacitive linear loads}

Consider two linear loads for the same topology of Figure 3, the first consisting of a $R=2 \Omega$ in series with an $L=0.01 \mathrm{H}$, and the second one $R=2 \Omega$ in series with a $C=0.001 \mathrm{~F}$; the supply voltage includes harmonics of orders 5 and 7 . Tables 5 and 6 present the results.

\begin{tabular}{cccc}
\hline & Two loads & Load 1 & Load 2 \\
\hline P1 (W) & $4.4590 \cdot 10^{4}$ & $2.2504 \cdot 10^{4}$ & $2.2086 \cdot 10^{4}$ \\
\hline P5 (W) & $7.4478 \cdot 10^{2}$ & $1.2859 \cdot 10^{1}$ & $7.3192 \cdot 10^{2}$ \\
\hline P7 (W) & $9.0580 \cdot 10^{1}$ & $7.7483 \cdot 10^{1}$ & $8.9805 \cdot 10^{1}$ \\
\hline PT (W) & $4.5425 \cdot 10^{4}$ & $2.2518 \cdot 10^{4}$ & $2.2908 \cdot 10^{4}$ \\
\hline PH (W) & $8.3536 \cdot 10^{2}$ & $1.3634 \cdot 10^{1}$ & $8.2172 \cdot 10^{2}$ \\
\hline
\end{tabular}

Table 5. Harmonic and total powers in the case 1. 


\begin{tabular}{cccc}
\hline & Two loads & Load 1 & Load 2 \\
\hline ITDD & 17.43 & 2.46 & 18.94 \\
\hline HPI & 0.00 & 0.00 & 0.00 \\
\hline NC & 7.34 & 12.74 & 20.58 \\
\hline NL & 7.34 & 0.00 & 18.08 \\
\hline
\end{tabular}

Table 6. PQ indices in the case 1.

Analysis of Table 6 shows how, in this case, only HPI rate indices identify the presence of linear loads in each measuring point. Although the HPI index identifies linear loads, however, then you will see that this index does not succeed when the system is unbalanced. The NL index can identify the RL load as linear but fails with RC load. The NC index fails to identify any such linear loads.

\subsection{Case 2: Non linear load and capacitive load}

Secondly, we have been considered a six-pulse controlled rectifier (load 1) in parallel with a linear load RC (load 2). This case is the same as above was used to obtain the waveforms presented in Figure 4. The table 7 includes the values obtained for the deferent indexes. In this case none of the identified indexes load 2 as linear load. The ITDD is the amplification of harmonics due to the presence of the capacitive load. Rates of identification of nonlinear loads HPI, NC, and NL, have high values for linear RC load.

\begin{tabular}{lccc}
\hline & Two loads & Load 1 & Load 2 \\
\hline ITDD & 28.90 & 33.78 & 56.49 \\
\hline HPI & 28.56 & 32.32 & 40.37 \\
\hline NC & 31.95 & 34.84 & 56.04 \\
\hline NL & 30.48 & 34.82 & 53.80 \\
\hline
\end{tabular}

Table 7. Index values with the presence of capacitor banks.

\section{Assessment of unbalance emission}

From the point of view of electromagnetic compatibility we distinguish between immunity and emission of a particular disturbance. The emission of imbalance consists of measuring the current phase sequence different from the positive sequence injected by an unbalanced 
load into the PCC. This section introduces the most common index to assess the imbalance emission.

\subsection{Conforming and nonconforming unbalance current}

Regarding the issue unbalance, for three-phase phase voltages $\mathrm{V}_{\mathrm{a}}, \mathrm{V}_{\mathrm{b}}$, and $\mathrm{V}_{\mathrm{c}}$, and line currents $I_{a}, I_{b}$, and $I_{c}$, symmetrical components at the fundamental frequency are considered, $V_{+}$, $\mathrm{V}-, \mathrm{V}_{0}, \mathrm{I}_{+}, \mathrm{I}-$ and $\mathrm{I}_{0}$. These are defined for the voltages by the following expressions:

$$
\begin{gathered}
\mathrm{V}_{-}=\frac{1}{3}\left(\mathrm{~V}_{\mathrm{a}}+\mathrm{a}^{2} \mathrm{~V}_{\mathrm{b}}+\mathrm{a} \mathrm{V}_{\mathrm{c}}\right) \\
\mathrm{V}_{0}=\frac{1}{3}\left(\mathrm{~V}_{\mathrm{a}}+\mathrm{V}_{\mathrm{b}}+\mathrm{V}_{\mathrm{c}}\right) \\
\mathrm{I}_{\mathrm{n}+}=\mathrm{I}_{+}
\end{gathered}
$$

Where complex number $a=\exp (j 2 \pi / 3)$. Similarly symmetrical components of currents are defined. The conforming current is the current that retains the same level of unbalance that the three-phase voltages, and coincides with the positive sequence current of the load current. Thus, the conforming current accomplishes all the active power and reactive power of positive sequence.

$$
Z_{+}=\frac{V_{+}}{I_{+}}
$$

The ratio between $\mathrm{V}_{+}$and $\mathrm{I}_{+}$determines the impedance to the positive sequence current

$$
\mathrm{I}_{\mathrm{n}-}=\frac{\mathrm{V}_{-}}{\mathrm{Z}_{+}}=\mathrm{I}_{+} \frac{\mathrm{V}_{-}}{\mathrm{V}_{+}}
$$

The negative sequence component and zero sequence component of the conforming current will be in the same proportion as the sequence components of voltage, that is,

$$
\begin{gathered}
I_{n 0}=\frac{V_{0}}{Z_{+}}=I_{+} \frac{V_{0}}{V_{+}} \\
I_{d+}=0
\end{gathered}
$$


The non conforming current is the current balance. In terms of symmetrical components,

$$
\begin{gathered}
\mathrm{I}_{\mathrm{d}-}=\mathrm{I}_{-}-\mathrm{I}_{\mathrm{n}-} \\
\mathrm{I}_{\mathrm{d} 0}=\mathrm{I}_{0}-\mathrm{I}_{\mathrm{n} 0} \\
i_{b l j}(t)=\sum_{k=1}^{n} \frac{\mathrm{V}_{k j}}{\mathrm{Z}_{\mathrm{ka}} \mid} \sqrt{2} \sin \left(2 \pi k f_{1} t+\left(\theta_{k j}-\varphi_{k j}\right)\right) \\
j=b, c
\end{gathered}
$$

Finally, the three phase values $\left(I_{d a}, I_{d b}, I_{d c}\right)$ are obtained from the inverse transformation of Fortescue. Although the author does not indicate a specific index of unbalance, we have considered the ratio of norm of non-conforming currents with respect to the norm of the real current, NC_unb.

\subsection{Balanced linear currents and unbalanced non-linear currents}

To define an index that takes into account the charge imbalance, be chosen as the reference phase current phase extracted with the minimum RMS value. For further analysis this phase will be considered as phase ' $a$ '. According to the procedure developed by the authors, [19], for estimating the parameters, it is assumed that the equivalent linear and balanced load is made up by three linear loads, balanced, equal to the estimated linear load for the phase 'a'. Thus, the current absorbed by the phases 'b' and ' $c$ ' for the same RL series load, estimated on phase 'a', are

$$
i_{u n l j}(t)=i_{j}(t)-i_{b l j}(t) \quad ; \quad j=b, c
$$

These currents are called "balanced linear currents" and only responsible for the unbalance and distortion is the delivery system. The difference between the measured actual current in each phase and the calculated ideal current is called "unbalanced non-linear current",

$$
i_{u j}(t)=i_{u n l j}(t)-i_{n l j}(t) \quad ; \quad j=b, c
$$

These currents expresses how the phases 'b' and 'c' differ from ideal reference conditions in terms of phase unbalance and distortion. On the other hand, it is possible to calculate nonlinear currents $i_{n l j}(\mathrm{t})(\mathrm{j}=\mathrm{b}, \mathrm{c})$ for phases 'b' and ' $\mathrm{c}$ ' in the manner provided in the previous 
section. Assuming that it is possible to separate the contribution of the distortion only by subtracting the nonlinear current $i_{n l j}(\mathrm{t})$ of $i_{\text {unlj }}(\mathrm{t})$ in the corresponding phase, then,

$$
\frac{\left\|i_{u}\right\|}{\left\|i_{n l}\right\|} \times 100(\%)
$$

These currents expresses how the phases ' $b$ ' and ' $c$ ' differ from ideal reference conditions only in terms of phase unbalance.

Once they have been introduced all these current components, it is possible to determine the degree of unbalance of a three phase load through the following definition of "unbalance current index",

$$
\begin{gathered}
\left\|i_{u}\right\|=\sqrt{\sum_{j=b, c} I_{u j}^{2}} \quad\left\|i_{n l}\right\|=\sqrt{\sum_{j=a, b, c} I_{n l_{j}}^{2}} \\
I_{u j}=\sqrt{\sum_{\forall k} I_{k u j}^{2}} \quad I_{n l j}=\sqrt{\sum_{\forall k} I_{k u l_{j}}^{2}}
\end{gathered}
$$

Where RMS values $I_{u j}$ and $I_{j}$ and norms of the set phase currents are defined as follow,

$$
V_{e}=\sqrt{\frac{V_{a b}^{2}+V_{b c}^{2}+V_{c a}^{2}}{9}}
$$

$\mathrm{I}_{\mathrm{kuj}}$ and $\mathrm{I}_{\mathrm{knlj}}$ are the RMS values of corresponding harmonic components of $i_{u j}(\mathrm{t})$ and $i_{n l j}(\mathrm{t})$. Defined (46) has the disadvantage that for linear unbalanced loads takes very high values, even infinite. So, here has changed their definition by making the rate against the norm of the real current.

Finally it is also possible to evaluate the harmonic distortion on a three-phase basis by combining the values of the three different rates of each phase in a single index defined as the ratio between the norm of non-linear currents and the norm of the load currents.

\subsection{Practical case: Digital simulation}

As a case study a three-phase nonlinear unbalanced system is considered. A set of three loads are connected at the PCC. The load 1 is a balanced three-phase topology formed by three single-phase rectifier with a RL branch into DC side. The load 2 is an unbalanced nonlinear load constituted by a single-phase rectifier with a RC branch into DC side in one phase and two resistors of different values in the remaining phases. Finally, the load 3 is a linear inductive and unbalanced load. Table 8 shows the total and harmonic powers, respec- 
tively, for each of the loads and for the whole of them. This time the harmonic power identifies the first two loads as non-linear and third load as linear.

\begin{tabular}{ccccc}
\hline & Three loads & Load 1 & Load 2 & Load 3 \\
\hline PT(W) & $3.5433 \times 10^{4}$ & $1.2740 \times 10^{4}$ & $4.2502 \times 10^{3}$ & $1.8443 \times 10^{4}$ \\
\hline PH(W) & -3.4792 & -2.4387 & -3.7266 & 2.6861 \\
\hline
\end{tabular}

Table 8. Harmonic and total active powers for unbalance loads.

Table 9 presents the usual distortion indices. They identify the sources of distortion except index HPI. This is based on the direction of power flows for each harmonic between network and load, and masks the power unbalance due to it. However, no information is provided on the issue of unbalance.

On the other hand, NC and NL indices identify the load 1 and the load 2 as non-linear, and the load 3 as linear.

\begin{tabular}{ccccc}
\hline & Three loads & Load 1 & Load 2 & Load 3 \\
\hline ITDD & 25.86 & 59.53 & 86.35 & 1.27 \\
\hline HPI & 25.97 & 39.67 & 86.56 & 35.59 \\
\hline NC & 18.75 & 40.72 & 73.64 & 0.50 \\
\hline NL & 16.80 & 34.02 & 83.41 & 0.20 \\
\hline
\end{tabular}

Table 9. $\mathrm{PQ}$ indices in the practical case with unbalance loads.

Table 10 presents the values of the unbalance indices ITDD ${ }^{+}, \mathrm{NC}_{-}$unb and UC. The first characterized the conformity of the current waveform of each load with the balanced sinusoidal waveform. The remaining indices identify unbalance responsability. Both identify the balanced load (load 1) and assign a value to unbalance loads.

\begin{tabular}{ccccc}
\hline & Three loads & Load 1 & Load 2 & Load 3 \\
\hline ITDD+ & 46.16 & 59.53 & 89.21 & 64.67 \\
\hline NC_unb & 26.71 & 0.27 & 30.27 & 47.14 \\
\hline UC & 41.19 & 0.29 & 25.38 & 68.99 \\
\hline
\end{tabular}

Table 10. Indices of unbalance in the practical case with asymmetrical conditions.

One issue to note is that NC_unb index is obtained by applying the symmetrical components, while the UC not, this could lead to it not identify a supply voltage of negative sequence. 


\section{Practical cases: Experimental results}

In the order to perform a validation of the results of simulations and verify the stability of the indices, has been built an experimental platform consists of three single-phase rectifiers connected in star and fed directly from the supply network. On the DC side of each rectifier has been connected a parallel RC branch, composed of a variable resistor and a capacitor $2200 \mu \mathrm{F}$, thus forming a nonlinear load, see Figure 5.

The measurement system consists of a data acquisition card (dspace-CP1104) and a signal conditioning system formed by three voltage sensors (LEM LV25-P) and three current sensors (LEM LA35-NP), thus the voltage and current signals are taken simultaneously, without introducing any phase change that could affect the accuracy of measurements. For data acquisition and processing, has developed a virtual instrument using Matlab and ControlDesk. This instrument stores the instantaneous values of each phase voltage and line current. The configuration of the virtual instrument was made following the recommendations EN 61 000-4-7 and EN 61 000-4-30, so that has been used window equal to five cycles of the fundamental component and a sampling frequency of $6400 \mathrm{~Hz}$, thus avoiding problems of aliasing and leakage errors.

To evaluate the theory and the results obtained in simulations has been carried out daily measurements along a day at regular intervals of one hour. In this way has been possible to evaluate the changes into indices for two different cases, a non lineal balanced load(case A) and another case with non lineal unbalanced load (case B). In both cases the supply system network was used for feeding the loads.

\subsection{Case A: Non linear and balanced load}

In this case the load connected to the DC side of the three rectifiers consists of a resistance of $120 \Omega$ in parallel with a capacitor $2200 \mu \mathrm{F}$. In Figure 6 (a) displays the voltage waveforms of the three phases whereas the Figure 6 (b) shows the waveforms of the currents taken from one of the measurements performed on the test system.

Table 11 shows the values of total active power $\left(\mathrm{P}_{\mathrm{T}}\right)$ consumed by the load on the three different measurements made throughout the session, as well as fundamental harmonic $\left(\mathrm{P}_{1}\right)$ and the values of total active harmonic power $\left(\mathrm{P}_{\mathrm{H}}\right)$.

\begin{tabular}{cccc}
\hline & M 3 & M 15 & M 21 \\
\hline PT (W) & 2134.00 & 2245.00 & 2210.00 \\
\hline P1 (W) & 2169.00 & 2288.90 & 2254.40 \\
\hline PH (W) & -35.02 & -43.85 & -44.41 \\
\hline
\end{tabular}

Table 11. Active powers of the main harmonics of the test system in Figure 4 with Non linear and balanced load (Case A). 
Due to variations in the supply voltage throughout the day, small variations are produced in the active power consumed by the load. Analysis of Table 11, it follows that, as corresponding to the nonlinearity of the load, the total active harmonic power is negative. Furthermore. it can be seen as the values of total active harmonic power $\left(\mathrm{P}_{\mathrm{H}}\right)$ are very small compared to the active power of fundamental harmonic. Moreover, as expected, there was a small value of VTDD throughout the day, about $3 \%$, while the value of ITDD is considerably higher, around $78 \%$. Figure 7 (a) shows the variations of VTDD throughout the day, whereas in Figure 7 (b) shows the variations of ITDD along test system and includes the results of the different rates to be evaluated.

\subsection{Case B: Non lineal and unbalanced load}

For unbalancing the load has changed the value of the resistances of each rectifier DC side, so that R1 $=80 \Omega, R 2=120 \Omega$ and $\mathrm{R} 3=484 \Omega$, while the capacitor is the same in the three loads, $2200 \mu \mathrm{F}$. In Figure 8 (a) shows the waveforms of voltages (supply network) whereas the figure 8 (b) shows the waveforms of currents resulting from the measurements taken.

Table 12 shows the values of total active power $\left(\mathrm{P}_{\mathrm{T}}\right)$ consumed by the load on the three measurements made throughout the session, as well as the values of total active harmonic power $\left(\mathrm{P}_{\mathrm{TH}}\right)$, and active power for the fundamental $\left(\mathrm{P}_{\mathrm{T} 1}\right)$.

Figure 9 shows how the measurement system includes all of the daily variation harmonic content and unbalance of the supply network voltage, whereas in Figure 9 (b) shows the daily trend of results unbalanced indices calculated for the same period.

\begin{tabular}{|c|c|c|c|c|c|c|c|c|c|}
\hline & \multicolumn{3}{|c|}{ M3 } & \multicolumn{3}{|c|}{ M15 } & \multicolumn{3}{|c|}{ M21 } \\
\hline & Phase L1 & Phase L2 & Phase L3 & Phase L1 & Phase L2 & Phase L3 & Phase L1 & Phase L2 & Phase L3 \\
\hline$P 1(W)$ & 993.43 & 714.46 & 194.32 & 1041.80 & 752.64 & 202.11 & 1037.50 & 751.76 & 200.94 \\
\hline PTH (W) & & -30.46 & & & 1958.80 & & & 1951.10 & \\
\hline PT (W) & & 1871.80 & & & 1996.60 & & & 1990.20 & \\
\hline PT1 (W) & & 1902.20 & & & -37.82 & & & -39.13 & \\
\hline
\end{tabular}

Table 12. Active powers of the main harmonics of the test system in Figure 4 with Non lineal and unbalanced load (Case B).

The experimental results confirm the simulation results on the information provided by the PQ indices. The measurements show that for a typical load balanced/unbalanced nonlinear connected to the supply network, all the indices analyzed included some variations to changes of TDD voltage within a reasonable range. In any case, the index NC_unb respect the degree of unbalance, is the index experienced less variations. This index is defined from 
the fundamental harmonic symmetrical components, and therefore undergone fewer changes for a given load.

\section{Discussion of the results and conclusions}

The strong presence of waveforms of voltage and current distorted and/or unbalanced in electric power systems has driven the need to determine the contribution to the deterioration of the PQ of consumers connected to supply networks. The method of the direction of power flow has been widely used to identify the locations of harmonic sources. However, this method is unable to solve this task in all situations. This procedure does not locate a source of distortion in the case that there are multiple harmonics sources connected to the PCC. To overcome this situation, other procedures have been introduced under the constraint of seeking a solution, based on the realization of measures in only one section of the PCC. These methods have introduced new indices as they are, HPI, NC and NL, that advance but which do not solve the problem in all situations. Thus:

- The method of direction of harmonic power flow fails when there is more than a nonlinear load connected to the same PCC. The measurement of $\mathrm{P}_{\mathrm{H}}$ can be positive at the terminals of a nonlinear load. This is because the harmonic powers up the sum of $\mathrm{P}_{\mathrm{H}}$ are the contribution of power flow of two opposing, in the sense from load to network and another from network to load. The result for each harmonic corresponds to the prevailing power flow. Therefore, for the identification of sources of distortion, $\mathrm{P}_{\mathrm{H}}$ is a not suitable indicator and would have to resort the study of the individual harmonics powers. However, this method is the only one that unambiguously identifies the linear loads, including those with capacitor banks for compensation.

- The index of harmonic phase, HPI, overcomes the drawbacks mentioned for the method of harmonic power direction, using the RMS values of current in one direction or another in the PCC. Moreover presents an adequate stability to changes in network distortion. However, result in errors for linear loads on systems with unbalanced and with the presence of the capacitor bank.

- The non-conforming current index, NC, while indicating the current component of the load presented to the voltage distortion front, is not useful from the practical point of view since it does not discriminate linear loads. In the case of the presence of capacitors is identified as distortion source. Moreover, this index shows more variation compared to other indices variations in the conditions of distortion of the mains.

- The non-linear current index, NL, it seems appropriate to characterize the current of a nonlinear load distorted. It also presents few variations to changes in the distortion of the mains voltage. However, the rate can result in errors in linear loads with different topologies including the RL parallel and/or capacitive branches.

Respect to the issues of unbalance loads produced by asymmetrical operation has been introduced ITDD+ index as a measure of non-conformity of the waveform of actual current 
with respect to the balanced sinusoidal waveform positive sequence. This index, as the ITDD, characterized the set of waveforms of a three phase system but will not identify unbalanced loads. Two indexes to locate unbalance are introduced: Current Unbalance index, UC and Non-Conforming to the unbalance, NC_unb. Both identify the loads that are sources of unbalance, however, the UC would not detect the case of a supply voltage of negative sequence.

In conclusion, in the text above have reviewed the potential problems associated with the assessment of power quality in electrical installations. In particular, we have introduced harmonic distortion and unbalanced to characterize the voltage and current waveforms. A comparative analysis of the most common indices was made. Thus, to determine responsible for the generation of distortion through measurements made in a single measurement section, none of the indexes given is capable of resolving the issue reliably. However, since in practice all loads are nonlinear, the NL is an appropriate index for assessing the distortion source because it is little affected by the imbalance and distortion. For the determination of the issue of unbalance NC_unb index has shown a good performance.

\section{Summary of indices}

Effective Voltage, $V_{e}=\sqrt{\frac{V_{a b}^{2}+V_{b c}^{2}+V_{c a}^{2}}{9}}$

Effective Current, $I_{e}=\sqrt{\frac{I_{a}^{2}+I_{b}^{2}+I_{c}^{2}}{3}}$

Voltage Total Harmonic Distortion, $V T H D_{\phi}=\sqrt{\frac{V_{\phi}^{2}-V_{\phi_{1}}^{2}}{V_{\phi_{1}}^{2}}}=\sqrt{\frac{V_{\phi_{2}}^{2}+V_{\phi_{3}}^{2}+V_{\phi_{4}}^{2}+\ldots+V_{\phi_{N}}^{2}}{V_{\phi_{1}}^{2}}}$

Current Total Harmonic Distortion, ITHD ${ }_{\phi}=\sqrt{\frac{I_{\phi}^{2}-I_{\phi_{1}}^{2}}{I_{\phi_{1}}^{2}}}=\sqrt{\frac{I_{\phi_{2}}^{2}+I_{\phi_{3}}^{2}+I_{\phi_{4}}^{2}+\ldots+I_{\phi_{N}}^{2}}{I_{\phi_{1}}^{2}}}$

Voltage Total Demand Distortion, $V T D D_{\phi}=\sqrt{\frac{V_{\phi}^{2}-V_{\phi_{1}}^{2}}{V_{\phi}^{2}}}=\sqrt{\frac{V_{\phi_{2}}^{2}+V_{\phi_{3}}^{2}+V_{\phi_{4}}^{2}+\ldots+V_{\phi_{N}}^{2}}{V_{\phi}^{2}}}$

Current Total Demand Distortion, ITDD ${ }_{\phi}=\sqrt{\frac{I_{\phi}^{2}-I_{\phi_{1}}^{2}}{I_{\phi}^{2}}}=\sqrt{\frac{I_{\phi_{2}}^{2}+I_{\phi_{3}}^{2}+I_{\phi_{4}}^{2}+\ldots+I_{\phi_{N}}^{2}}{I_{\phi}^{2}}}$

Three-Phase Total Harmonic Distortion of Current and Voltage, $I T H D_{3 \phi}=\sqrt{\frac{I_{e}^{2}-I_{e 1}^{2}}{I_{e 1}^{2}}} ; \operatorname{VTHD}{ }_{3 \phi}=\sqrt{\frac{V_{e}^{2}-V_{e 1}^{2}}{V_{e 1}^{2}}}$ 
Three-Phase Total Demand Distortion of Current and Voltage, $I T D D_{3 \phi}=\sqrt{\frac{I_{e}^{2}-I_{e 1}^{2}}{I_{e}^{2}}} ; V T D D_{3 \phi}=\sqrt{\frac{V_{e}^{2}-V_{e}^{2}}{V_{e}^{2}}}$

Unbalance Three-Phase Total Demand Distortion of Voltage and Current, $V T D_{3 \phi}^{+}=\sqrt{\frac{V_{e}^{2}-V_{e 1+}^{2}}{V_{e}^{2}}} ; \quad \operatorname{ITDD_{3\phi }^{+}}=\sqrt{\frac{I_{e}^{2}-I_{e 1+}^{2}}{I_{e}^{2}}}$

The Harmonic Phase Index, HPI $=\frac{\left\|I_{L}\right\|}{\left\|I_{S}\right\|} \times 100(\%)$

Non Collinear Index, NC $=\frac{I_{d}}{I} \times 100(\%)$

Unbalanced Non Collinear Index, NC_unb $=\frac{\left\|i_{d}\right\|}{\|i\|} \times 100(\%)$

Non Linear Index, $N L=\frac{I_{n L a}}{I_{a}} \times 100(\%)$

Unbalanced Current Index, $U C=\frac{\left\|i_{u}\right\|}{\left\|i_{n l}\right\|} \times 100(\%)$

\section{Acknowledgments}

This work is part of the project "Measurements system to assessment the sources of imbalance and harmonic distortion in electric distribution networks", DPI2010-17709, sponsored by the Ministry of economy and competitiveness, Government of Spain.

\section{Author details}

Patricio Salmerón Revuelta and Alejandro Pérez Vallés

Departament of Electrical Engineering, E.T.S.I, Huelva University, Spain

\section{References}

[1] A. Menchetti, R. Sasdelli, Measurement Problems in Power Quality Improvement, ETEP, vol. 4, No. 6, Nov/Dec, 1994.

[2] A. Ferrero, A. Menchetti, R. Sasdelli, Measurement of the Electric Power Quality and Related Problems, ETEP, Vol. 6, No. 6, November/December 1996. 
[3] G.T. Heydt, E. Gunther, 1996 "Post-measurement processing of electric power quality data", IEEE Trans. on Power Delivery, Vol. 11, No. 4.

[4] G.T. Heydt, W.T. Jewel, 1998 "Pitfall of electric power quality indices", IEEE Trans. on Power Delivery, Vol. 13, No. 2.

[5] D.D. Sabin, D.L. Brooks, A. Sundaram, 1999, "Indices for assessing harmonic distortion from power quality measurements: definitions and benchmark data", IEEE Trans. on Power Delivery, Vol. 14, No. 2.

[6] R. Sasdelli, G. Del Gobbo, G. Iuculano, 2000 “Quality management for electricity as a processed material". IEEE Trans. on Instrumentation and Measurement, Vol. 49, No. 2 .

[7] F.J. Alcántara, P. Salmerón. A New Technique for Unbalanced Current and Voltage Measurement with Neural Networks. IEEE Trans. On Power Delivery, Vol. 20 No: 2, May 2005.

[8] P. Salmerón, R. S. Herrera, J. Prieto, A. Pérez. New Distortion and Unbalance Indices based on Power Quality Analyzer Measurements. IEEE Transaction on Power Delivery, Vol. 24, No. 2, April 2009, pp: 501-507.

[9] L. S. Czarnecki, T. Swietlicki, Powers in Nonsinusoidal Networks: Their Interpretation, Analysis, and Measurement, IEEE Trans. on Instr. and Measurement, Vol. 39, No. 2, April 1990.

[10] P. H. Swart, M. J. Case, J. D. Van Wyk, On Techniques for Localization of Sources Producing Distorsion in Electric Power Networks, ETEP, vol. 4, No. 6 Nov/Dec 1994

[11] P. H. Swart, J. D. Van Wyk, M. J. Case, On Techniques for Localization of Sources Producing Distorsion in Three-Phase Networks, ETEP, vol. 6, No. 6 Nov/Dec 1996.

[12] Srinivasan, K. On Separating Customer and Supply Side Harmonic Contributions, IEEE Trans. on Power Delivery, vol. 11, No. 2, April 1996.

[13] E. Thunberg y L. Söder. A Norton Approach to Distribution Network Modeling for Harmonic Studies. IEEE Transaction on Power Delivery, Vol. 14, No. 1, January 1999

[14] A. P. J. Rens y P.H. Swart. On Techiques for the Localization of Multiple Distortion Sources in Three-Phase Networks: Time Domain Verification. ETEP Vol. 11, No. 5, September/ October 2001.

[15] C. Muscas, Assessment of Electrical Power Quality: Indices for Identifying Disturbing Loads, ETEP, Vol. 8, No. 4, July/August 1998.

[16] J.P.V. Du Toit, J.H.C. Pretorius y W.A. Cronje. Non Linear Load Identification under Non-Sinusoidal Conditions. Sixth International Workshop on Power Definitions and Measurenments under Non-Sinusoidal Conditions, Milano, October 13-15, 2003. 
[17] W. Xu, X. Liu y Y. Liu. An Investigation on the Validity of Power-Direction Method for Harmonic Source Determination. IEEE Transations on Power Delivery, Vol. 18, No. 1, January 2003.

[18] Ch. Chen, X. Liu, D. Koval, W. Xu y T. Tayjasanant. Critical Impedance Method- A New Detecting HarmonicSources Method in Distribution Systems. IEEE Transations on Power Delivery, Vol. 19, No. 1 January 2004.

[19] A. Dell'Aquila, M. Marinelli, V. G. Monopoli y P. Zanchetta. New Power-Quality Assessment Criteria for Supply Under Unbalanced and Nonsinusoidal Conditions. IEEE Transactions on Power Delivery, Vol. 19, No. 3, July 2004.

[20] Cataliotti and V. Consetino, A New Measurement Method for the Detection of Harmonic Sources in Power Systems Base on the Approach of the IEEE Std. 1459-2000, IEEE Transactions On Power Delivery, Vol.25, No. 1, pp. 332-340, January 2010.

[21] Reyes S. Herrera, Patricio Salmerón. Harmonic disturbance identification in electrical systems with capacitor banks. Electric Power Systems Research, Volume 82, Issue 1, January 2012, Pages 18-26.

[22] J.E. Farach, W.M. Grady y A. Arapostathis. An Optimal Procedure for Placing Sensors and Estimating the Locations of Harmonic Sources in Power Systems. IEEE Transations on Power Delivery, Vol. 8, No. 3, July 1993.

[23] L. Critaldi, A. Ferrero y S. Salicone. A Distributed System for Electric Power Quality Measurement. IEEE Transaction on instrumentation and Measurement, Vol. 51, No. 4, August 2002.

[24] C. Muscas, L. Peretto, S. Sulis, R. Tinarelli, Investigation on Multipoint Measurement Techniques for PQ Monitoring, IEEE Trans. Instrum. Meas. Vol. 51, pp. 1684, oct. 2006.

[25] N. Locci, C. Muscas, S Sulis, On the Measurement of Power-Quality Indexes for Harmonic Distortion in the Presence of Capacitors, IEEE Transactions on Instrumentation and Measurement, Vol 56, Issue 5, pp.1871 - 1876, Oct. 2007.

[26] Definitions for the measurement of electric power quantities under sinusoidal, nonsinusoidal, balanced, or unbalanced conditions, IEEE Std 1459-2000, January 2000. 